Case Report

\title{
Successful Rotational Atherectomy for an Angulated Calcified Lesion in an Anomalous Right Coronary Artery Using the "Mother-and-Child" Technique
}

\author{
Manabu Ogita $\mathbb{D}^{1},{ }^{1}$ Satoru Suwa, ${ }^{1}$ Taketo Sonoda, ${ }^{1}$ Shuta Tsuboi, ${ }^{1}$ Katsumi Miyauchi, ${ }^{2}$ and \\ Hiroyuki Daida ${ }^{2}$ \\ ${ }^{1}$ Department of Cardiology, Juntendo University Shizuoka Hospital, Izunokuni, Japan \\ ${ }^{2}$ Department of Cardiovascular Medicine, Juntendo University Graduate School of Medicine, Tokyo, Japan \\ Correspondence should be addressed to Manabu Ogita; m-ogita@sa2.so-net.ne.jp
}

Received 29 September 2017; Accepted 25 December 2017; Published 14 January 2018

Academic Editor: Hajime Kataoka

Copyright (C) 2018 Manabu Ogita et al. This is an open access article distributed under the Creative Commons Attribution License, which permits unrestricted use, distribution, and reproduction in any medium, provided the original work is properly cited.

\begin{abstract}
Percutaneous coronary intervention (PCI) involving the anomalous coronary artery is challenging with respect to difficulty in achieving stable catheterization. Rotational atherectomy (RA) can facilitate severely calcified lesions to improve stent delivery and stent expansion; however, its utility in tortuous and angulated coronary arteries is limited with difficulty in delivery of the RA burr. The mother-and-child technique is effective for complex PCIs with increased backup force for device delivery in such complicated cases. We report a case of successful rotational atherectomy using the "mother-and-child" technique with a Dio thrombus aspiration catheter for an angulated calcified lesion in an anomalous origin of the right coronary artery.
\end{abstract}

\section{Introduction}

Coronary artery anomalies are a diverse group of congenital disorders that are found in $5 \%$ of the population [1]. Anomalous origin of the right coronary artery (RCA) accounts for $10 \%$ of all anomalous coronary artery cases [2]. Percutaneous coronary intervention (PCI) involving the anomalous coronary artery is challenging with respect to selection of the guide catheter, insufficient backup force, and device delivery. The mother-and-child technique is useful for improving backup support and delivery of stents in such lesions [3]. Rotational atherectomy (RA) can facilitate lesion and stent expansion in severely calcified lesions especially in patients undergoing hemodialysis [4]; however, its utility is limited in tortuous and angulated coronary arteries, with an increased risk of vessel perforation and difficulty in delivery of the RA burr. We describe a case of successful rotational atherectomy for an angulated calcified lesion in the anomalous right coronary artery using the "mother-and-child" technique.

\section{Case Presentation}

A 46-year-old man with end-stage renal disease due to diabetic nephropathy was referred to our institution for percutaneous coronary intervention (PCI) for a severe calcified lesion in an anomalous right coronary artery. He had been undergoing hemodialysis three times a week for 3 years. Since patients with diabetic ESRD are at a high risk for coronary artery disease, coronary computed tomography angiography (CTA) had been performed at his previous hospital visit, which revealed multivessel disease. Coronary angiography (CAG) showed severe stenosis in the midportion of the left anterior descending (LAD) artery and a chronic total occlusion (CTO) in the proximal portion of the right coronary artery with an anomalous origin. Hence, PCI of the LAD lesion was first performed, and a bioresorbable polymer sirolimus-eluting stent (Ultimaster $3.0 \times 38 \mathrm{~mm}$ and $3.0 \times 33 \mathrm{~mm}$, TERUMO, Tokyo, Japan) was successfully implanted at the previous hospital. Staged PCI for CTO of the RCA was performed with successful wire 


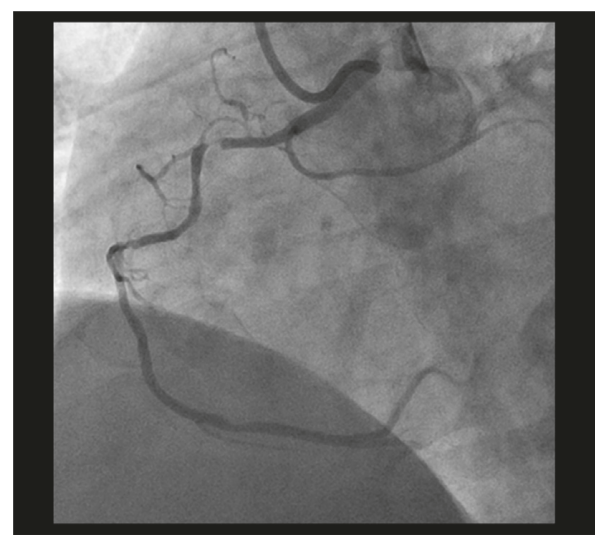

FIGURE 1: The left anterior oblique coronary angiography view showed severe calcified stenosis of the proximal segment of the right coronary artery (RCA) with anomalous origin.

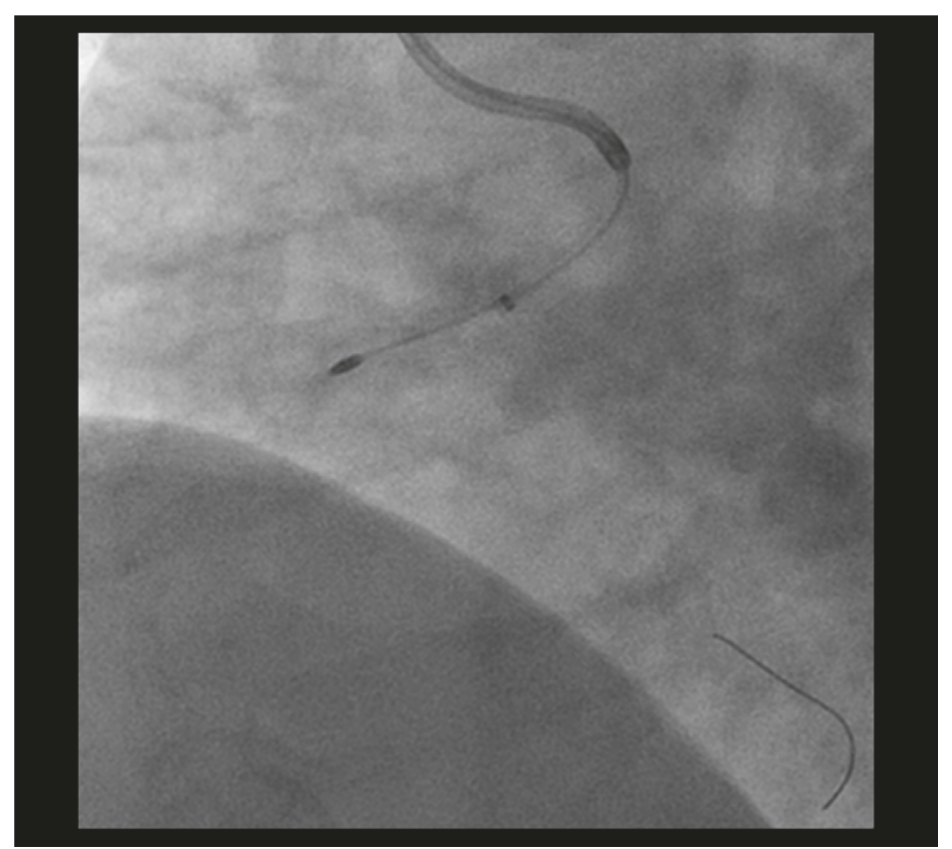

Figure 2: Advancement of a $1.5 \mathrm{~mm}$ burr through the Dio thrombus aspiration catheter.

crossing and recanalization. However, since subsequent standard balloon angioplasty was unsuccessful, he was referred to our hospital for PCI of the anomalous RCA.

CAG demonstrated residual severely calcified stenosis in the proximal RCA with patent antegrade coronary flow (Figure 1). We selected right femoral access with a long sheath using a 7 Fr Amplatz left 2 guiding catheter (Asahi Intecc, Tokyo, Japan) in consideration of size up to a $2.0 \mathrm{~mm}$ burr. A SION blue wire (Asahi Intecc) was advanced into the distal RCA using a microcatheter (Caravel, Asahi Intecc) and intravascular ultrasonography (IVUS; OptiCross, Boston Scientific Corp., MA, USA). Based on the IVUS findings, which indicated severe circumferential calcification with a minimal luminal diameter larger than $1.25 \mathrm{~mm}$, we decided to perform rotational atherectomy with a $1.5 \mathrm{~mm}$ burr (Boston Scientific Corp., MA, USA). For this, the first wire was exchanged for an extrasupport RotaWire (Boston
Scientific) using the microcatheter. To obtain greater backup force, a Dio thrombus aspiration catheter (Goodman Co. Ltd., Aichi, Japan) was inserted into the RCA. The burr was successfully delivered to the anomalous RCA with dynaglide because we had difficulty advancing the burr manually. Halfway ablation with a $1.5 \mathrm{~mm}$ burr at $200,000 \mathrm{rpm}$ was attempted several times, taking care not to advance the burr beyond the angle [5], although subsequent CAG and IVUS did not show the effective lesion modification after ablation. Then, we switched to balloon angioplasty with a $2.5 \mathrm{~mm}$ high-pressure balloon (NC Emerge $2.5 \times 12 \mathrm{~mm}$, Boston Scientific) and a $2.5 \mathrm{~mm}$ cutting balloon (Flextome $2.5 \times 10 \mathrm{~mm}$, Boston Scientific), but it was not enough to dilate the lesion. Finally, we switched back to rotational atherectomy and were subsequently able to pass the burr beyond the angulated calcified lesion without complications (Figure 2) and confirmed a crack using IVUS. Following 


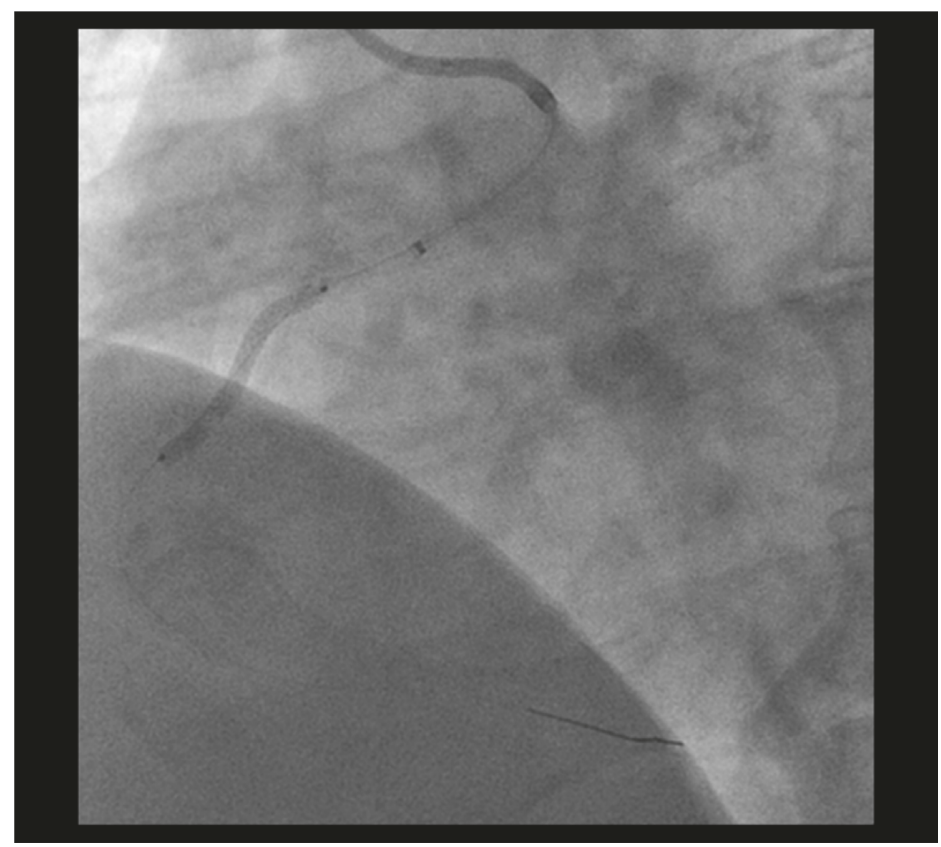

FIgURE 3: Successful stent deployment within the lesion.

effective dilation with a $3.0 \mathrm{~mm}$ high-pressure balloon (CELUSUS $3.0 \times 8 \mathrm{~mm}$, NIPRO, Osaka, Japan), we deployed a bioresorbable polymer everolimus-eluting stent (Synergy $2.5 \times 24 \mathrm{~mm}$, Boston Scientific) (Figure 3). The stent was additionally dilated with a noncompliance balloon (CELUSUS $3.0 \times 8 \mathrm{~mm}$, NIPRO, Osaka, Japan). A final coronary angiogram showed adequate and favorable dilatation of the culprit lesion (Figure 4).

\section{Discussion}

We report a case of successful rotational atherectomy for an angulated calcified lesion in an anomalous right coronary artery using the "mother-and-child" technique. PCI for the anomalous RCA can be associated with difficulty in achieving stable catheterization, leading to reduced backup support and device delivery failure. Rotational atherectomy (RA) can facilitate lesion and stent expansion in severely calcified lesions, although its utility is limited in tortuous and angulated coronary arteries, with an increased risk of complications. Appropriate guiding catheter selection is a key step to ensure successful PCI in such cases.

The mother-and-child technique is effective for complex PCIs, as it increases the backup force through deep catheterization of the target vessel and facilitates device delivery in tortuous or calcified lesions. Several mother-and-child catheters have been introduced in clinical practice (Table 1). The GuideLiner catheter is a guide extension catheter with the monorail system which permits rapid exchange and now is, hence, increasingly used [6]. Only a few cases have been previously reported of use of the mother-and-child technique to deliver rotational atherectomy burrs. Vo et al. first reported the use of the GuideLiner catheter to deliver rotational atherectomy burrs in tortuous vessels [7]. Costanzo et al. also reported GuideLiner-facilitated rotational

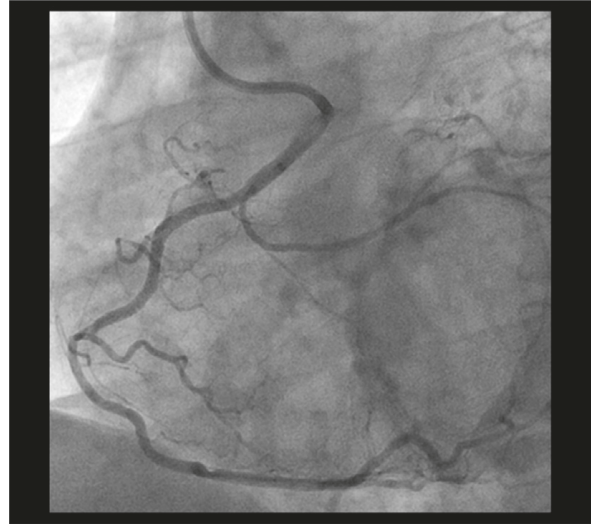

FIGURE 4: Final angiographic results after stent implantation and after dilatation.

atherectomy in calcified right coronary arteries [8]. However, the mother-and-child technique for rotablation has not been well established. To the best of our knowledge, this is the first report of RA using the mother-and-child technique in an RCA with anomalous origin.

In our case, we selected the conventional mother-child catheter, the 5 Fr Dio aspiration catheter for the following reasons. First, the 5 Fr Dio thrombus aspiration catheter has the RX-type inner catheter which facilitates smooth delivery and deep insertion of the outer catheter into the anomalous RCA. The relatively large inner lumen size of the outer catheter $(1.51 \mathrm{~mm})$ can provide the strong backup force for device delivery. Second, there is a concern that the rapid exchange transition zone of GuideLiner can lead to less backup support, difficulty in delivering the burr, and increase in the risk of burr entrapment. Thus, we thought that the Dio thrombus aspiration catheter can provide stronger backup support in our patient with rather complicated 
TABLE 1: Design comparison of the guide extension catheter available in Japan and Dio thrombus aspiration catheter.

\begin{tabular}{|c|c|c|c|c|}
\hline & Guidezilla II (Boston Scientific) & GuideLiner V3 (Lifeline) & Guideplus (NIPRO) & Dio (Goodman) \\
\hline Sizes & $6 \mathrm{Fr}, 7 \mathrm{Fr}, 8 \mathrm{Fr}$, and $6 \mathrm{Fr}$ long & $5.5 \mathrm{Fr}, 6 \mathrm{Fr}, 7 \mathrm{Fr}$ & $6 \mathrm{Fr}$ & $5 \mathrm{Fr}$ \\
\hline \multirow[t]{4}{*}{ Inner lumen } & & $1.30 \mathrm{~mm}(5.5 \mathrm{Fr})$ & $1.30 \mathrm{~mm}(6 \mathrm{Fr})$ & $1.51 \mathrm{~mm}(5 \mathrm{Fr})$ \\
\hline & $1.45 \mathrm{~mm}(6 \mathrm{Fr})$ & $1.42 \mathrm{~mm}(6 \mathrm{Fr})$ & & \\
\hline & $1.60 \mathrm{~mm}(7 \mathrm{Fr})$ & $1.57 \mathrm{~mm}(7 \mathrm{Fr})$ & & \\
\hline & $1.83 \mathrm{~mm}(8 \mathrm{Fr})$ & $1.80 \mathrm{~mm}(8 \mathrm{Fr})$ & & \\
\hline \multirow[t]{4}{*}{ Outer lumen } & & $1.60 \mathrm{~mm}(5.5 \mathrm{Fr})$ & $1.50 \mathrm{~mm}(6 \mathrm{Fr})$ & $1.72 \mathrm{~mm}(5 \mathrm{Fr})$ \\
\hline & $1.45 \mathrm{~mm}(6 \mathrm{Fr})$ & $1.70 \mathrm{~mm}(6 \mathrm{Fr})$ & & \\
\hline & $1.60 \mathrm{~mm}(7 \mathrm{Fr})$ & $1.90 \mathrm{~mm}(7 \mathrm{Fr})$ & & \\
\hline & $2.11 \mathrm{~mm}(8 \mathrm{Fr})$ & $2.16 \mathrm{~mm}(8 \mathrm{Fr})$ & & \\
\hline Guide segment length & $25 \mathrm{~cm} / 40 \mathrm{~cm}$ (6 Fr long) & $25 \mathrm{~cm}$ & $25 \mathrm{~cm}$ & \\
\hline Working length & $150 \mathrm{~cm}$ & $150 \mathrm{~cm}$ & $145 \mathrm{~cm}$ & $124 \mathrm{~cm}$ \\
\hline Coating & Z-glide (hydrophilic) & Silicon wipe (hydrophilic) & Hydrophilic & Hydrophilic \\
\hline
\end{tabular}

anatomy. Komatsu et al. reported successful PCI in an anomalous RCA using the Dio thrombus aspiration catheter [9]. This catheter allows not only stent delivery but also rotating burr delivery to severe calcified lesions in anomalous RCAs and can be a useful adjunctive device for rotational atherectomy. It is essential for operators to position the burr distal to the Dio thrombus aspiration catheter to prevent potential damage and shear of the child catheter. In summary, the "mother-and-child" technique is useful for rotational atherectomy in anomalous angulated RCAs. Rotablation with a Dio thrombus aspiration catheter is a potential therapeutic strategy in such complicated cases.

\section{Conclusion}

We report a case of successful rotational atherectomy using the "mother-and-child" technique with a Dio thrombus aspiration catheter for an angulated calcified lesion in an anomalous origin of the right coronary artery.

\section{Conflicts of Interest}

The authors declare that there are no conflicts of interest regarding the publication of this paper.

\section{Acknowledgments}

The authors thank all the nurses and technicians of the cath lab in Juntendo Shizuoka Hospital for their professional contributions and assistance.

\section{References}

[1] P. Angelini, "Coronary artery anomalies: an entity in search of an identity," Circulation, vol. 115, no. 10, pp. 1296-1305, 2007.

[2] O. Yamanaka and R. E. Hobbs, "Coronary artery anomalies in 126,595 patients undergoing coronary angiography," Catheterization and Cardiovascular Diagnosis, vol. 21, no. 1, pp. 28-40, 1990.

[3] S. Takahashi, S. Saito, S. Tanaka et al., "New method to increase a backup support of a 6 French guiding coronary catheter," Catheterization and Cardiovascular Interventions, vol. 63 , no. 4 , pp. 452-456, 2004.
[4] M. I. Tomey, A. S. Kini, and S. K. Sharma, "Current status of rotational atherectomy," JACC: Cardiovascular Interventions, vol. 7, no. 4, pp. 345-353, 2014.

[5] K. Sakakura, Y. Taniguchi, M. Matsumoto, H. Wada, S. I. Momomura, and H. Fujita, "How should we perform rotational atherectomy to an angulated calcified lesion?," International Heart Journal, vol. 57, no. 3, pp. 376-379, 2016.

[6] C. Cola, F. Miranda, B. Vaquerizo, A. Fantuzi, and J. Bruguera, "The GuideLiner ${ }^{\mathrm{TM}}$ catheter for stent delivery in difficult cases: tips and tricks," Journal of Interventional Cardiology, vol. 24, no. 5, pp. 450-461, 2011.

[7] M. Vo, K. Minhas, M. Kass, and A. Ravandi, "Novel use of the GuideLiner catheter to deliver rotational athetectomy burrs in tortuous vessels," Case Reports in Cardiology, vol. 2014, Article ID 594396, 5 pages, 2014.

[8] P. Costanzo, K. Aznaouridis, S. Hoye, and A. Alahmar, "GuideLiner-facilitated rotational atherectomy in calcified right coronary artery: the "child" makes the difference," JACC: Cardiovascular Interventions, vol. 9, no. 5, pp. e47-e48, 2016.

[9] T. Komatsu, I. Yaguchi, and T. Yufu, "Successful percutaneous coronary intervention of an anomalous right coronary artery with high anterior takeoff using a DIO thrombus aspiration catheter," Journal of Invasive Cardiology, vol. 24, no. 9, pp. E185-E187, 2012. 


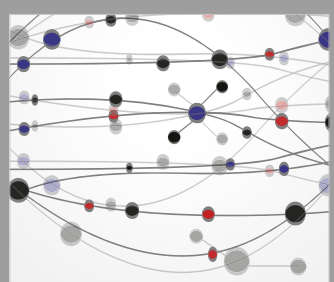

The Scientific World Journal
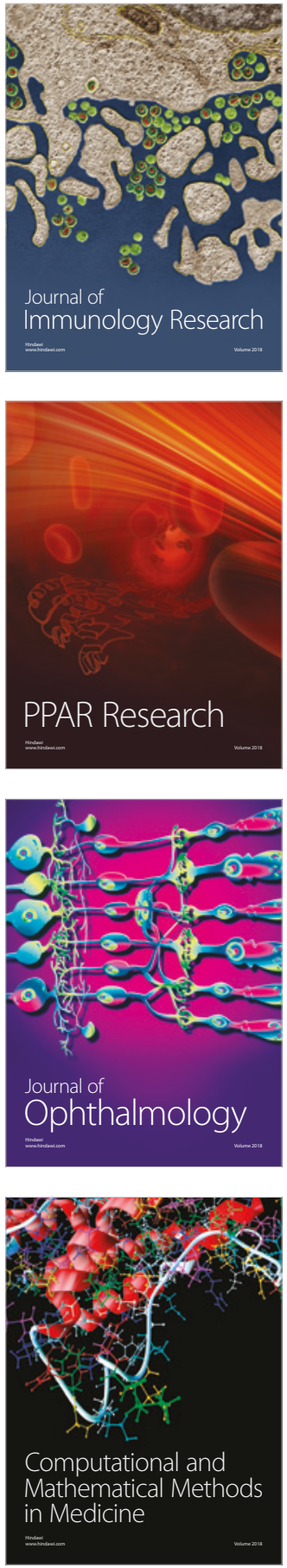

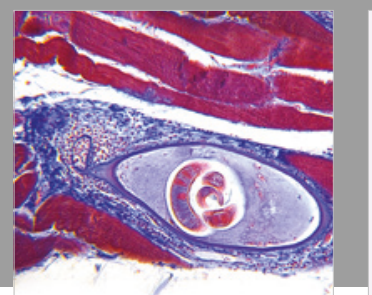

Gastroenterology Research and Practice

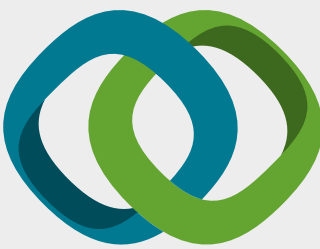

\section{Hindawi}

Submit your manuscripts at

www.hindawi.com
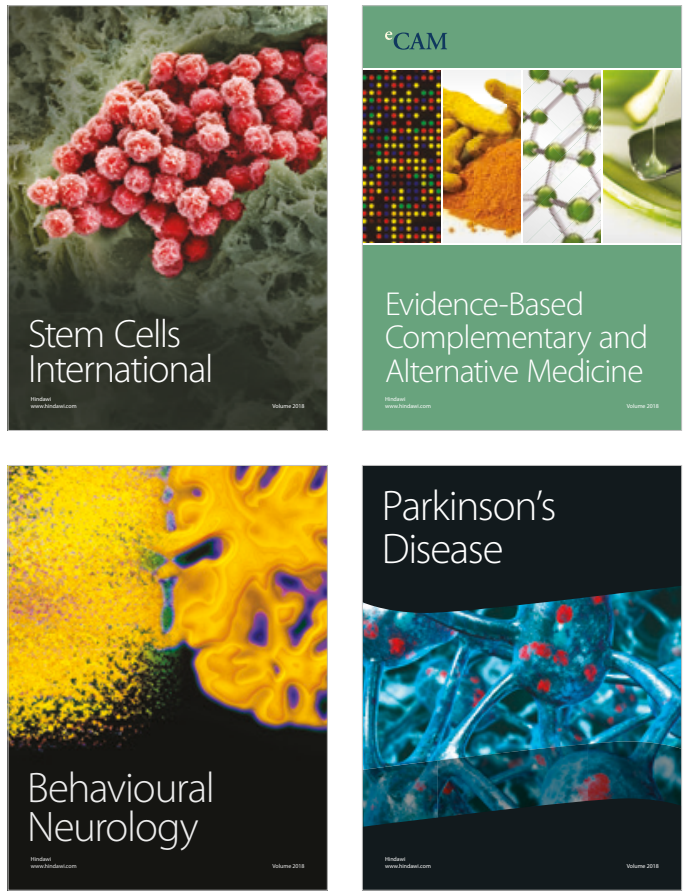

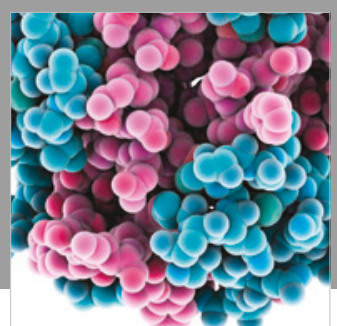

ournal of

Diabetes Research

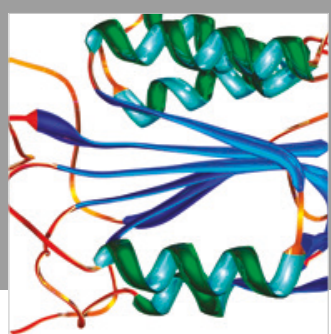

Disease Markers
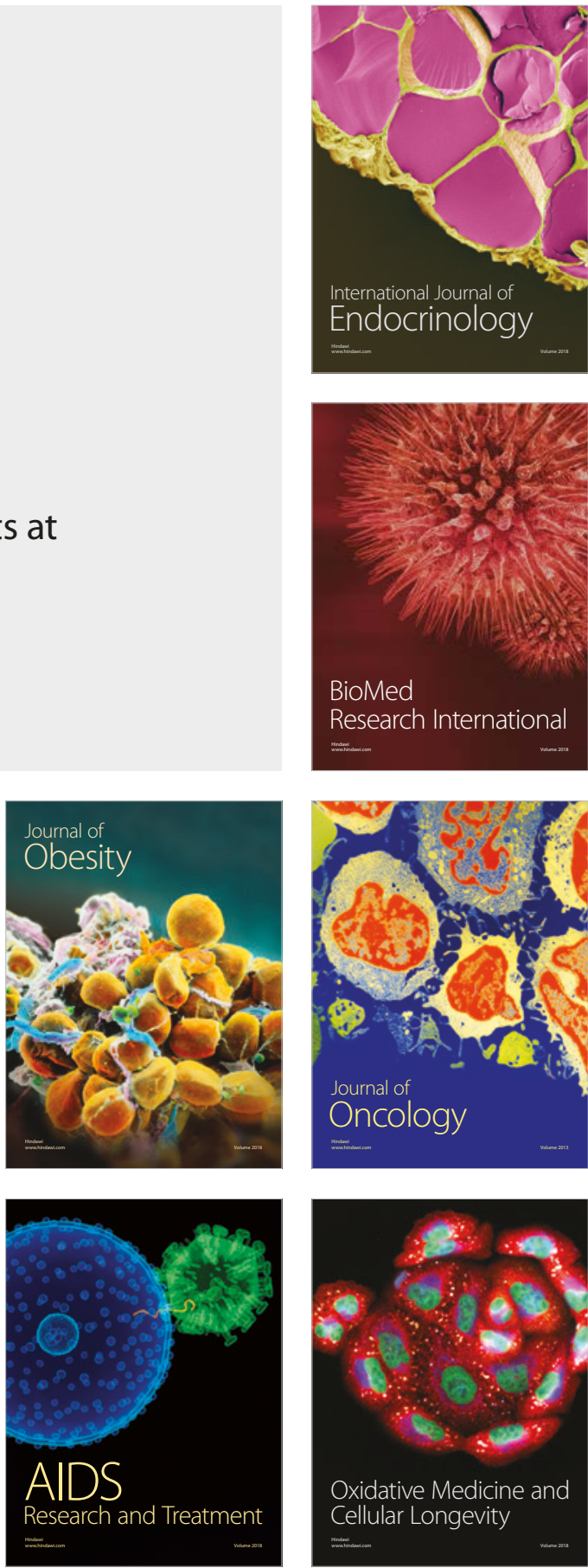